\title{
Physiologically-Based IVIVC of Azithromycin
}

\author{
Nasir M. Idkaidek ${ }^{1, *}$, Naji Najib², Isam Salem², Jamal Jilani ${ }^{3}$ \\ ${ }^{1}$ College of Pharmacy, Petra University, Amman, Jordan \\ ${ }^{2}$ International Pharmaceutical Research Center, Amman, Jordan \\ ${ }^{3}$ College of Pharmacy, Jordan University of Science and Technology, Irbid, Jordan \\ *Corresponding author: nidkaidek@uop.edu.jo
}

Received August 21, 2014; Revised December 08, 2014; Accepted December 10, 2014

\begin{abstract}
Aim of the study is to establish physiologically-based in vitro in vivo correlation (IVIVC) of azithromycin, a biopharmaceutics classification system (BCS) class II drug (high permeability/ low solubility). In vitro dissolution was done using USP apparatus II in pH 6 phosphate buffer at $50 \mathrm{rpm}$. In vivo pharmacokinetic study was done on 28 healthy humans after IRB and Jordan FDA approvals. Plasma sampling was collected up to 72 hours. Non compartmental analysis was done using Kinetica program V 5. Physiologically based IVIVC was conducted using linear IVIVC module of SimCYP program V 13. Physiological parameter of effective intestinal permeability was optimized along with IVIVC calculations. IVIVC dissolution prediction matched in vivo profile with $1 \%$ and $7.7 \%$ prediction errors for $\mathrm{AUC}_{72}$ and Cmax, indicating proper physiologically based IVIVC. This is in agreement with IVIVC expectation for class II drugs according to (BCS) when in vitro dissolution rate is similar to in vivo dissolution rate. This is important for drug formulators to predict in vivo bioavailability from in vitro dissolution, which can save time and money.
\end{abstract}

Keywords: azithromycin, IVIVC, SimCYP, Intestinal Permeability

Cite This Article: Nasir M. Idkaidek, Naji Najib, Isam Salem, and Jamal Jilani, "Physiologically-Based IVIVC of Azithromycin.” American Journal of Pharmacological Sciences, vol. 2, no. 6 (2014): 100-102. doi: 10.12691/ajps-2-6-1.

\section{Introduction}

Azithromycin is a semi-synthetic antibiotic belonging to the macrolide subgroup of azalides and is similar in structure to erythromycin [1]. Azithromycin has an apparent advantage over erythromycin in that it reaches higher concentration intracellularly, thus increasing its efficacy and duration of action. Oral absorption of azithromycin is rapid but is inhibited by food, which also decreases the maximum plasma concentration. Distribution throughout the body is extensive. Azithromycin exhibits significant intracellular penetration and concentrates within fibroblasts and phagocytes. As a result, tissue levels are significantly higher than plasma concentrations. However, CNS penetration is poor. Peak plasma concentrations occur at about 2 hours after oral administration. The drug is eliminated largely in feces, following excretion into the bile, with less than $10 \%$ excreted in the urine. The half-life of elimination of azithromycin has been reported to be variable and can reach 70 hours, which is partially explained by its extensive tissue uptake and slow tissue release. The disposition of azithromycin from serum is a biphasic process, exhibiting a short tissue distribution phase followed by a longer elimination phase [2,3,4,5]. Azithromycin appears to demonstrate a time-dependent versus time-accumulation profile in breast milk [6]. In children, once-daily administration of azithromycin resulted in sustained systemic exposure to the drug [7]. In vitro dissolution is a very important test that must pass before going into in vivo studies. In vitro dissolution media are to be optimized in order to mimic in vivo environment, and hence can be used as a surrogate for in vivo studies under certain conditions [8].

The purpose of present study is to develop a successful Physiologically-Based IVIVC model for azithromycin, that can predict in vivo profile with minimum error.

\section{Materials and Methods}

\subsection{In Vitro Drug Dissolution}

The USP II rotating paddle apparatus was used with a stirring rate of $50 \mathrm{rpm}$, and maintained at $37^{\circ} \mathrm{C}$. 6 tested 15 ml-samples (Zithromax susp. Batch \# 021600) were placed in the dissolution media of $900 \mathrm{ml}$ phosphate buffer at $\mathrm{pH} 6.0 \mathrm{ml}$. Aliquot samples were withdrawn from the dissolution medium at 15, 30, 45 and 60 minutes and were immediately replaced by the same volume of fresh medium. The dissolution samples were filtered using $0.45 \mu \mathrm{m}$ millipore filter paper and assayed for azithromycin content.

\subsection{In Vivo Human Study}

plasma samples were collected at $0,0.0 .33,0.66,1,1.5$, 2, 2.5, 3, 3.5, 4, 4.5, 5, 6, 8, 10, 12, 24, 48 and 72 hours after $500 \mathrm{mg}$ oral dosing of azithromycin (Zithromax susp. 
Batch \# 021600) to 28 healthy humans. Plasma pharmacokinetic parameters were calculated by non compartmental analysis method using Kinetica V5. SimCYP program V13 was used for estimation of effective intestinal permeability and IVIVC modeling.

\subsection{Drug Marker Analysis}

All plasma samples were deep freezed until assayed by a validated LC-MS assay method. Intra-day CV was $10.17 \%$, inter-day accuracy and precision\% ranges were 92.8-100.94 and 6.97-10.03\%. Linear range was 1 - 200 $\mathrm{ng} / \mathrm{ml}$.

\subsection{Data Analysis}

\subsubsection{Calculated Pharmacokinetic Parameters}

Individual pharmacokinetic parameters for drug concentration in plasma samples were calculated by noncompartmental analysis (NCA) using Kinetica program. Pharmacokinetic parameters were area under the concentration curves to last collection time $\left(\mathrm{AUC}_{0 \rightarrow 72}\right)$, maximum measured concentration $\left(\mathrm{C}_{\max }\right)$ and time to maximum concentration $\left(\mathrm{T}_{\max }\right)$.

\subsubsection{IVIVC Analysis}

IVIVC tool was used along with effective permeability $\left(\mathrm{P}_{\text {eff }}\right)$ optimization, in order to match in vivo plasma profile. This was done by Nelder-Mead algorithm of Parameter Estimation module using SimCYP program [9]. Nelder-Mead method, which is also called downhill simplex, is a commonly used nonlinear optimization algorithm. This was done by searching for the best parameter values that produce plasma concentration that matches the actual plasma concentration at the same time. The objective function is the weighted sum of squared differences of observed and model predicted values. Polar surface area (PSA) was used first, using SimCYP, to predict initial estimate of $\mathrm{P}_{\text {eff }}$. All other physicochemical factors used in calculations such as $\log \mathrm{P}, \mathrm{MW}$ and fu were obtained from literature and were kept constant during the minimization processes. In vitro dissolution rate, in vivo clearance and volume of distribution were input from actual dissolution and plasma profiles.

\section{Results and Discussion}

Azithromycin mean in vitro and IVIVC dissolution profiles were presented in Figure 1 and Figure 2. Figure 1 showed that physiologically-based IVIVC dissolution profile is closer to bioavailability (F) profile, obtained from in vivo data, than in vitro dissolution profile. This was due to inclusion of permeability factor along with IVIVC, which is not the case in in-vitro profile as per USP method. It is worthwhile noting that azithromycin bioavailability reached around $60 \%$, which is in agreement with literature value of $57 \%$ [2]. Moreover, Figure 2 clearly showed that In-vitro and IVIVC dissolution profiles are strongly correlated $(\mathrm{R}=1)$. On the other hand, mean in vivo and IVIVC predicted plasma profiles shown in Figure 3 were in close agreement which indicates good fitting and good model prediction.
Table 1 showed that IVIVC predicted pharmacokinetics parameters of AUC and $\mathrm{C}_{\max }$ are close to actual ones, with prediction error of less than 10\%. However, Tmax was much longer in actual profile than in IVIVC prediction, which can be due to gastric empting in vivo that is not accounted for in IVIVC. This is in agreement with IVIVC expectation for class II drugs according to (BCS) where in vitro dissolution rate is similar to in vivo dissolution rate [10].

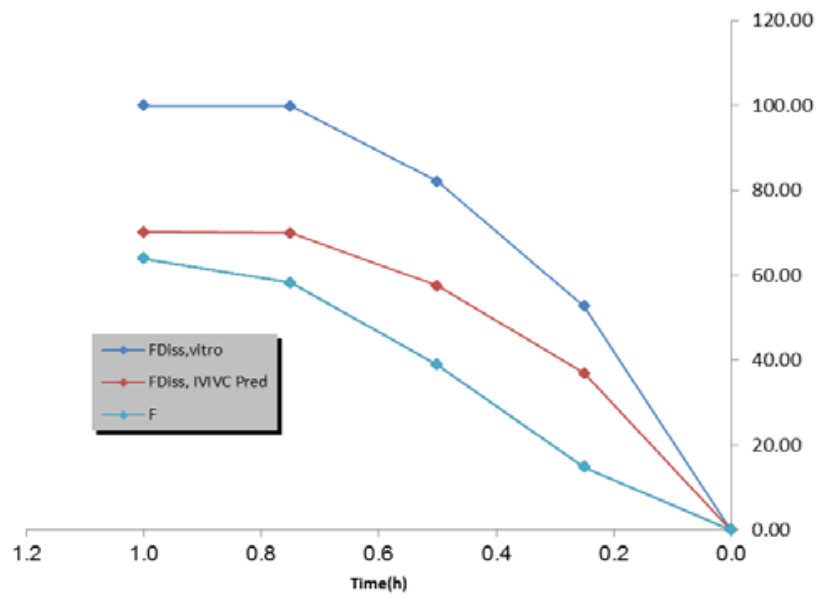

Figure 1. Azithromycin mean in vitro, IVIVC dissolution and Bioavailability profiles

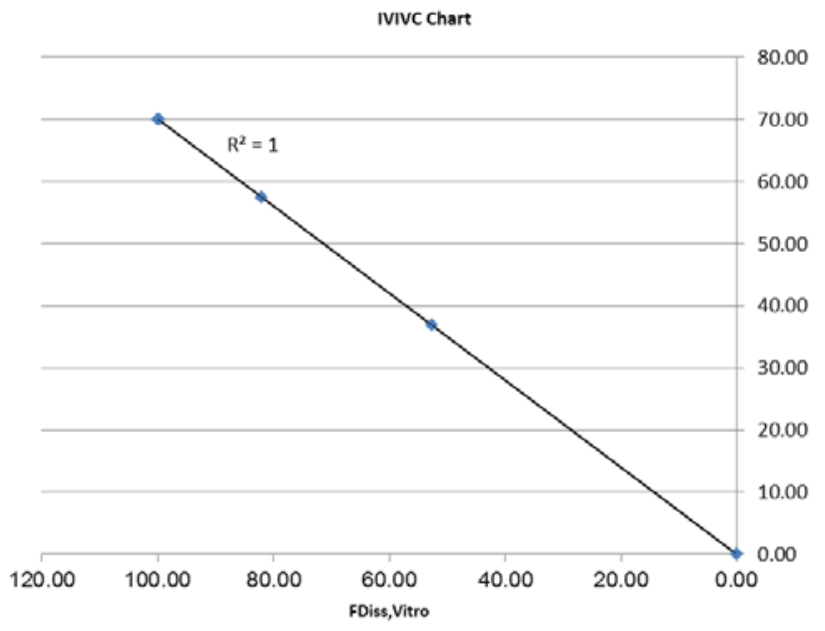

Figure 2. In vitro dissolution versus IVIVC dissolution

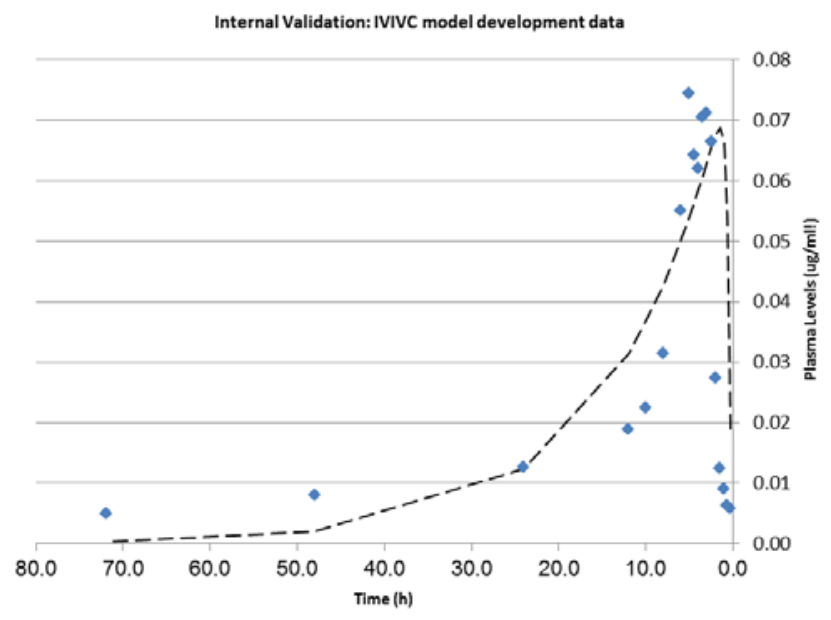

Figure 3. Plasma mean concentrations (ng/ml) after $500 \mathrm{mg}$ oral dose of azithromycin susp to 28 healthy humans, and IVIVC Prediction profile 
Table 1. Observed pharmacokinetic parameters of azithromycin as obtained from mean plasma profile, IVIVC Predictions and Prediction Errors

\begin{tabular}{|c|c|c|c|}
\hline Parameter & Observed & IVIVC Predicted & $\%$ PE \\
\hline AUC0-72 (ng.hr/ml) & 1051.2 & 1039.9 & 1.07 \\
\hline Cmax (ng/ml) & 74.4 & 68.7 & 7.7 \\
\hline Tmax (hr) & 4.7 & 1.2 & 74.5 \\
\hline
\end{tabular}

\section{Conclusions}

In summary, this study clearly shows that IVIVC dissolution prediction matched in vivo profile with $1 \%$ and $7.7 \%$ prediction errors in AUC and Cmax, indicating proper physiologically based IVIVC. This is in agreement with IVIVC expectation for class II drugs according to (BCS) since in vitro dissolution rate of azithromycin is similar to in vivo dissolution rate. This is important for drug formulators to predict in vivo bioavailability from in vitro dissolution, which can save time and money.

\section{Acknowledgment}

This research was funded by University of Petra and International Pharmaceutical Research Center. Kinetica was used under academic license from Innaphase Ltd, France (Lic. \# K 201009). SimCYP program was used under academic license from SimCYP Ltd, Sheffield, UK (Lic. \# CLCLID - AKDI - LEEE - FECI).

\section{References}

[1] Azithromycin data sheet: http://www.medsafe.govt.nz/profs/Datasheet/z/Zithromaxtabsusp. pdf

[2] Wildfeuer A, Laufen $H$, Leiold $M$ et al, Comparison of the pharmacokinetics of three-day and five-day regimens of azithromycin in plasma and urine. J Antimicrob. Chemother. 1993; 31: 51-56.

[3] Schentag J J \& Batlow CH. Tissue-directed pharmacokinetics. Am. J. Med. 1999; 91 (suppl. 3A): 5S-11S.

[4] Peters DH, Friedel HA, McTavish D. Azithromycin: A review of its antimicrobial activity, pharmacokinetic properties and clinical efficacy. Drugs. 1992; 44:750-99.

[5] Lalak NJ, Morris DL. Azithromycin clinical pharmacokinetics. Clin Pharmacokinet. 1993; 25: 370-4.

[6] Kelsey JJ, Moser LR, Jennings JC, Munger MA. Presence of azithromycin breast milk concentrations: a case report. Am J Obstet Gynecol. 1994; 170: 1375-6.

[7] Nahata MC, Koranyi KI, Gadgil SD, Hilligoss DM, Fouda HG, Gardner MJ Pharmacokinetics of azithromycin in pediatric patients after oral administration of multiple doses of suspension. Antimicrob Agents Chemother. 1993; 37: 314-6.

[8] J. B. Dressman, G. L. Amidon, C. Reppas, and V. P. Shah, Dissolution testing as a prognostic tool for oral drug absorption: immediate release dosage forms, Pharm. Res., 1998, 15 (1): 11-22.

[9] Jamei M, Turner D, Yang J, Neuhoff S, Polak S, RostamiHodjegan A and Tucker G. Population-based mechanistic prediction of oral drug absorption. AAPS J. 2009 Jun; 11 (2): 22537.

[10] Chi-Yuan Wu and Leslie Z. Benet. Predicting Drug Disposition via Application of BCS: Transport/Absorption/Elimination Interplay and Development of a Biopharmaceutics Drug Disposition Classification System. Pharmaceutical Researh 2005 January; 22 (1): 11-23. 